\title{
Surgical correction of aortic valve insufficiency after left ventricular assist device implantation
}

\author{
B. Zane Atkins, MD, ${ }^{\mathrm{a}}$ Zubair A. Hashmi, MD, ${ }^{\mathrm{b}}$ Asvin M. Ganapathi, MD, ${ }^{\mathrm{b}}$ J. Kevin Harrison, MD, ${ }^{\mathrm{c}}$ \\ G. Chad Hughes, MD, ${ }^{\mathrm{b}}$ Joseph G. Rogers, MD, ${ }^{\mathrm{c}}$ and Carmelo A. Milano, $\mathrm{MD}^{\mathrm{b}}$
}

Objectives: New-onset aortic insufficiency (AI) can be encountered after instituting mechanical circulatory support and seems more common and severe with continuous flow (CF) left ventricular assist devices (LVADs) compared with pulsatile devices. Treatment algorithms for de novo, post-LVAD AI have not been well defined. In the present report, we have described 6 patients who underwent aortic valve surgery for new-onset postLVAD AI.

Methods: From 2005 to 2011, 271 patients underwent LVAD implantation. Of these LVADs, 225 were CF devices (203 HeartMate II devices, Thoratec Corp, Pleasanton, Calif; and 22 HVAD devices, HeartWare Intl, Inc, Framingham, Mass). The patients were examined for new-onset severe AI requiring surgical intervention.

Results: During follow-up, 6 CF LVAD patients developed new, severe AI that was accompanied by heart failure. After medical therapy had failed, 4 patients underwent redo sternotomy for aortic valve procedures (1 bioprosthetic valve replacement, 1 Dacron patch closure, and 2 aortic valve repairs), and 2 patients underwent transcatheter aortic valve procedure, with 1 requiring revision by open surgery for aortic valve replacement. Of the 6 patients, 5 experienced significant improvement in functional capacity and symptoms. One patient died postoperatively secondary to multiorgan failure and sepsis.

Conclusions: Surgical treatment of post-LVAD AI with aortic valve oversewing or leaflet repair or by bioprosthetic aortic valve replacement is effective at restoring functional capacity for CF LVAD patients who develop symptomatic, severe AI and can be performed safely with good results. Various transcatheter approaches to these difficult problems are also available and offer less invasive alternatives to conventional surgery. (J Thorac Cardiovasc Surg 2013;146:1247-52)

A shortage of donor hearts for transplantation and recent improvements in mechanical circulatory support technologies have substantially increased the enthusiasm for implantation of left ventricular assist devices (LVADs). ${ }^{1}$ Although the long-term results with continuous flow (CF) LVAD therapy have been superior to those with pulsatile devices, ${ }^{2}$ some difficulties previously encountered with pulsatile devices remain problematic with CF devices. For example, native heart valve pathologic entities such as significant mitral stenosis, tricuspid regurgitation, and aortic insufficiency (AI) can prevent restoration of normal hemodynamics with LVAD support. Therefore, concomitant valvular procedures could be required at LVAD implantation. ${ }^{3}$

\footnotetext{
From the Division of Cardiothoracic Surgery, ${ }^{\mathrm{a}}$ Department of Surgery, University of California-Davis, Sacramento, Calif; and Division of Cardiovascular and Thoracic Surgery, ${ }^{b}$ Department of Surgery, and Division of Cardiology, ${ }^{\mathrm{c}}$ Department of Medicine, Duke University Medical Center, Durham, NC.

Disclosures: Dr Milano has received compensation from Thoratec Corporation for assistance with training programs; Dr Rogers serves as a consultant to Thoratec Corporation; and Dr Hughes serves as a consultant for W.L. Gore, Inc, and Terumo Corporation. The other authors have nothing to disclose with regard to commercial support.

Received for publication April 3, 2013; revisions received May 15, 2013; accepted for publication May 30, 2013; available ahead of print July 18, 2013

Address for reprints: Asvin M. Ganapathi, MD, Duke University Hospital, DUMC

Box 3443, Durham, NC 27710 (E-mail: asvin.ganapathi@duke.edu).

$0022-5223 / \$ 36.00$

Copyright (c) 2013 by The American Association for Thoracic Surgery

http://dx.doi.org/10.1016/j.jtcvs.2013.05.019
}

In addition, it has been previously recognized that newonset $\mathrm{AI}$ is frequently encountered during prolonged LVAD support, just as was first reported with pulsatile LVADs. ${ }^{4-6}$ The development of new, significant AI subsequent to instituting CF LVAD therapy has now been reported..$^{7-9}$ However, recent studies have suggested that CF LVAD support might be associated with more rapid progression of $\mathrm{AI}$ than that with pulsatile devices. ${ }^{10-12}$ Furthermore, at least 1 study has correlated de novo AI with worse long-term outcomes after LVAD support. ${ }^{12}$ Despite increasing awareness of the complications associated with AI during LVAD support, the treatment strategies for these patients have not been well defined. Specifically, given the increased risks accompanying reoperation and the inherent heterogeneity in the underlying valvular pathologic features, it is uncertain when surgical correction should be undertaken and what technique should be used to correct AI in this unique patient population. Certainly, the severity of valvular insufficiency, clinical status (symptoms of heart failure), and previous aortic valve surgery will influence the clinical decisions in this challenging population. To highlight the importance of these issues, we have described 6 cases (5 HeartMate II, Thoratec Corp, Pleasanton, Calif; and 1 HVAD, HeartWare Intl, Inc, Framingham, Mass) of surgical correction of AI after CF LVAD implantation. 


\section{Abbreviations and Acronyms \\ $\mathrm{AI}=$ aortic insufficiency \\ AVR $=$ aortic valve replacement \\ $\mathrm{CF}=$ continuous flow \\ $\mathrm{LVAD}=$ left ventricular assist device}

\section{METHODS}

After local institutional review board approval, we reviewed the records of 271 patients with end-stage heart failure who had undergone LVAD implantation at a single institution (2005-2011). Of the LVAD devices, 225 were CF LVADs (203 HeartMate II and 22 HVADs). At LVAD insertion, all patients underwent transesophageal echocardiography to evaluate the native cardiac valves and exclude intracardiac shunts. Routine patient follow-up at our institution consisted of transthoracic echocardiography every 6 months. During transthoracic echocardiography, attention was given to AI, with medical treatment of systemic blood pressure reduction used for any AI that is moderate or less. During this period, 5 patients underwent a concomitant aortic valve procedure at LVAD implantation for significant AI. Subsequently, 6 patients developed new, significant AI in the postoperative period requiring surgical intervention (Table 1), and we present these cases. The surgical care in each case was specifically tailored to the pathologic features present and overall patient health.

\section{RESULTS}

\section{Case 1}

A 75-year-old man underwent HeartMate II LVAD for ischemic cardiomyopathy as destination therapy. At 6 months postoperatively, he returned with new-onset, severe $\mathrm{AI}$ and increased pump power and pulsatility index. Percutaneous repair with an 18-mm Amplatzer PI Muscular ventricular septal defect occluder (AGA Medical, Plymouth, Minn) positioned at the level of the aortic annulus (Figure 1,A) was initially attempted; however, the device migrated into the left ventricle, prompting concerns for LVAD inflow cannula interference and causing severe AI (Figure 1,B). The patient underwent urgent redo sternotomy for ventricular septal defect occluder retrieval and aortic valvuloplasty due to incomplete central coaptation of central leaflets, using the technique described by Park et al. ${ }^{13}$ However, significant residual AI was identified after separation from cardiopulmonary bypass, and subsequent bioprosthetic aortic valve replacement (AVR) was performed with an uneventful recovery.

\section{Case 2}

A 70-year-old man with nonischemic cardiomyopathy secondary to rheumatic heart disease and remote bioprosthetic AVR underwent destination LVAD implantation with a HeartMate II. Four years later, he presented with refractory heart failure and severe bioprosthetic AI. Because of his poor underlying medical condition, he underwent off-label, transcatheter insertion of a Melody Transcatheter Pulmonary Valve (Medtronic, Minneapolis, Minn; Figure 2) within the previous bioprosthetic valve. Although residual, mild AI was present at the conclusion of the procedure, the patient was discharged from the hospital and experienced functional improvement.

\section{Case 3}

A 73-year-old woman with suspected doxorubicininduced cardiomyopathy underwent HeartMate II insertion as destination therapy. Trivial AI was evident preoperatively (Figure 3); however, 1 month later, she experienced heart failure symptoms, progressing to cardiogenic shock. Echocardiography revealed new-onset severe AI and severe tricuspid insufficiency. Despite aggressive medical therapy, the patient remained unstable and underwent emergent redo sternotomy. Significant prolapse of the noncoronary cusp was noted after aortotomy, bioprosthetic AVR, and tricuspid valve annuloplasty was performed. Notably, the patient also had left ventricular and intrapump thrombus, and a pump exchange was performed. Unfortunately, the patient developed sepsis and renal failure and died 6 weeks later.

\section{Case 4}

A 67-year-old man underwent HeartMate II LVAD insertion for ischemic cardiomyopathy. Approximately 1 year later, he developed new, recurrent heart failure symptoms, with echocardiographic findings of new severe AI with a concurrent increased pump power and pulsatility index. $\mathrm{He}$ underwent redo sternotomy with cardiopulmonary bypass. Marked thickening of all 3 leaflets and commissural fusion was noted. Consequently, Dacron patch closure of the aortic valve was performed. The patient recovered well, with functional improvement despite significant preoperative debilitation.

\section{Case 5}

A 67-year-old woman underwent destination therapy with an HVAD for ischemic cardiomyopathy. Four months later, she presented with recurrent heart failure and newonset, severe AI. Redo sternotomy revealed scarring and retraction of the left coronary cusp. Several sutures were placed through the nodules of Arantius to elevate the retracted cusp to the level of the non- and right coronary cusps, resolving the valvular insufficiency. The patient did well postoperatively and demonstrated functional improvement.

\section{Case 6}

A 41-year-old woman presented with acute myocardial infarction and underwent emergency coronary artery bypass grafting. She developed postcardiotomy shock and required extracorporeal membrane oxygenation, with subsequent urgent placement of a HeartMate II LVAD. Two years later, she presented with severe heart failure and hemolysis. Echocardiography revealed newly diagnosed moderate-to-severe AI. After medical management failed, 
TABLE 1. Patient demographics and characteristics

\begin{tabular}{lccclcl}
\hline Pt. no. & Age (y) & $\begin{array}{c}\text { Pathologic } \\
\text { features }\end{array}$ & LVAD type & \multicolumn{1}{c}{ Pre-LVAD TEE } & $\begin{array}{c}\text { Interval from VAD } \\
\text { implantation to severe AI } \\
\text { presentation (mo) }\end{array}$ & Procedure performed \\
\hline 1 & 75 & ICM & HMII & No AI & 6 & Open AVR \\
2 & 70 & NICM & HMII & $\begin{array}{c}\text { Bioprosthetic aortic valve, } \\
\text { trivial AI }\end{array}$ & 48 & Percutaneous valve-in-valve \\
3 & 73 & NICM & HMII & Trivial AI & 1 & Open AVR; VAD pump exchange \\
4 & 67 & ICM & HMII & Mild AI & 12 & Open Dacron patch closure \\
5 & 67 & ICM & HVAD & Trace AI & 4 & Open Park sutures \\
6 & 43 & ICM & HMII & No AI & 28 & Open Park sutures; VAD pump \\
& & & & & & exchange \\
\hline
\end{tabular}

Pt. no., Patient number; LVAD, left ventricular assist device; TEE, transesophageal echocardiography; ICM, ischemic cardiomyopathy; HMII, HeartMate II assist device (Thoratec Corp, Pleasanton, Calif); AI, aortic insufficiency; AVR, aortic valve replacement; NICM, nonischemic cardiomyopathy; HVAD, HeartWare Intl, Inc assist device (Framingham, Mass); VAD, ventricular assist device.

she underwent redo sternotomy, and a restricted right coronary cusp with commissural fusion was noted. Valve repair with interrupted polypropylene sutures, to elevate the right coronary cusp to the level of the left and noncoronary cusps was performed. Because of the complicating history of hemolysis with the potential for pump thrombosis, the patient also underwent LVAD pump replacement. The patient did well postoperatively and was discharged to home.

Overall, surgical correction of AI was accomplished using several different techniques, which were individually selected according to unique features of the case. In all 6 cases, echocardiography demonstrated correction of severe AI, and, with the exception of 1 patient, all experienced significant functional recovery. Two transcatheter treatments were used, and in 1 of the cases, a catheter-based, valve-in-valve strategy was successful.

\section{DISCUSSION}

For patients with end-stage heart failure, CF LVAD support as a bridge to transplantation or as destination therapy has been shown to improve survival, functional status, quality of life, and exercise capacity. ${ }^{2,14-16}$ Furthermore, compared with pulsatile devices, CF LVADs have greater durability. ${ }^{2,16}$ Given the extended survival of patients supported with CF LVAD therapy, previously uncommon disorders are now being seen with increasing frequency. ${ }^{17,18}$ For example, CF LVAD therapy has been implicated in the development of acquired AI, acquired von Willebrand syndrome with mucosal bleeding, and other issues associated with the physiology of nonpulsatile blood flow. ${ }^{17,19}$ As Frazier ${ }^{14}$ has suggested, clinicians treating patients with an CF LVAD must be adept at investigating and managing these relatively new disorders.

In the setting of LVAD therapy, AI leads to blood volume recirculation, inadequate left ventricular unloading, decreased systemic perfusion, and increased LVAD pump work. $^{20,21}$ These effects essentially produce clinical heart failure with progressive dyspnea and reduced functional status, and 1 study demonstrated patients developing AI after LVAD therapy had decreased survival. ${ }^{12}$ The propensity for developing AI during LVAD support appears to correlate with the duration of LVAD support, ${ }^{10}$ and the progression of AI might be greater with CF devices than with pulsatile devices. ${ }^{10-12}$ Furthermore, increased CF LVAD pump speeds result in greater left ventricular unloading and reduces pulsatility, creating near constant retrograde forces at the level of the sinuses of Valsalva and loss of normal systolic valvular relaxation. ${ }^{17,22,23}$ Progression of postoperative AI might correlate with the infrequency of aortic valve opening. ${ }^{10-13}$ A previous study in a nearly identical cohort of patients at our institution demonstrated that $11 \%$ of patients progressed to moderate or severe AI after LVAD implantation. Of these, all patients with moderate AI were able to be treated medically, but those with severe AI required surgical intervention. ${ }^{9}$ Regardless of the specific contributing factors, partial fusion and restriction of the aortic valve leaflets have been described as the anatomic changes during CF LVAD therapy that lead to AI. ${ }^{21-23}$ At least 4 of our 6 patients displayed these pathologic changes. It is unclear whether these pathologic changes affect the bioprosthetic valves. It is noteworthy that in patient 2 , the pre-existing bioprosthetic valve did not deteriorate within the same period as the native valves of the other 4 patients in our series.

The primary nonsurgical treatment of de novo $\mathrm{AI}$ in $\mathrm{CF}$ LVAD patients has been treatment of hypertension to reduce left ventricular and LVAD afterload and has been our standard therapy in this setting..$^{9,24,25}$ In all 6 patients in the present series, medical therapy was attempted, including afterload reduction, diuretics, and inotropes. In all 6 patients, these conservative measures failed to stabilize the patient and resolve the symptoms. Therefore, conservative measures are likely to be ineffective in the setting of severe AI and CF LVAD support. Notably, 1 of the 6 patients (patient 3) had an initial delay in surgical intervention and subsequently became quite unstable, providing an additional argument for expeditious surgical intervention. 

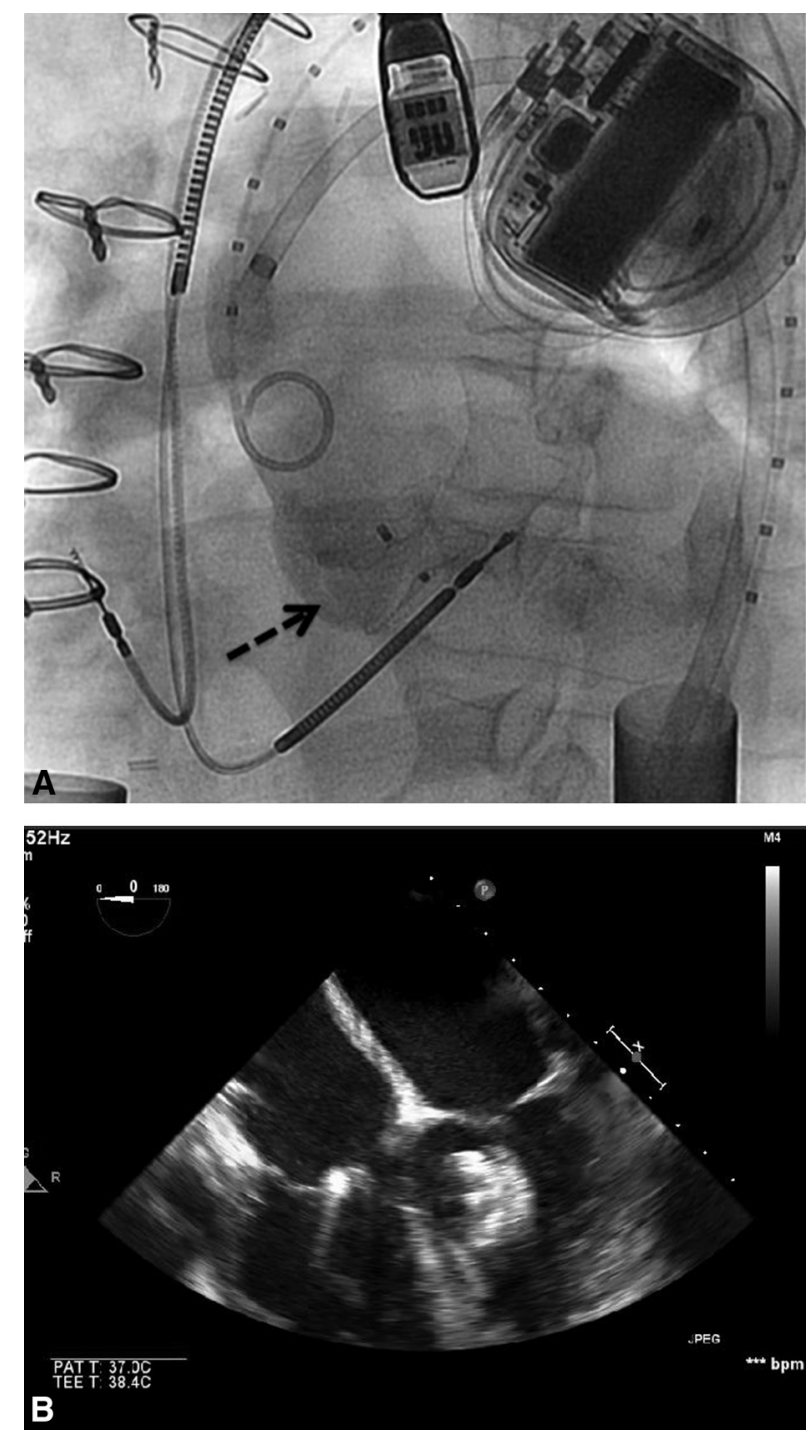

FIGURE 1. A, An 18-mm AMPLATZER PI Muscular ventricular septal defect Occluder was initially used to treat aortic regurgitation presenting 6 months after left ventricular assist device implantation. The ventricular septal defect occluder can be seen inferior to the pigtail catheter (dashed arrow). B, Despite the initial success, the ventricular septal defect occluder had migrated several days later (seen on echocardiography) and was associated with recurrent severe aortic insufficiency, which prompted open aortic valve replacement.

Redo sternotomy after LVAD implantation represents a major intervention with risks for right ventricular damage or failure and hemorrhage. Nevertheless, for patients who develop severe AI, open surgical correction might provide a survival advantage, given the lack of proven, successful alternative treatments. This approach of oversewing the aortic valve with a modified Park stitch represents our current practice guidelines in the setting of de novo severe AI. In 1 single-institution experience, long-term survival was shown to be significantly better among LVAD patients who underwent AI repair compared with LVAD patients
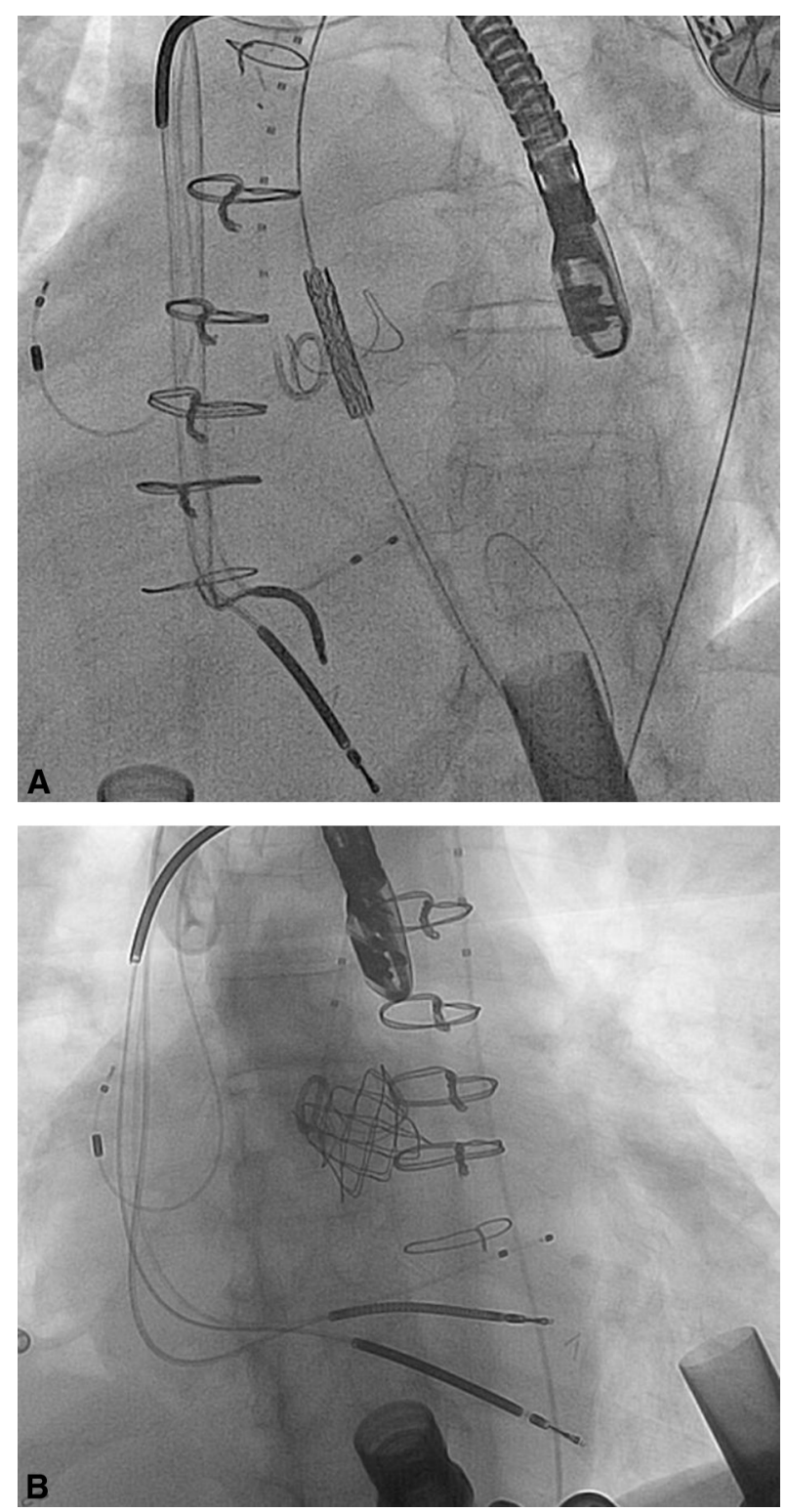

FIGURE 2. A, A transcatheter approach by way of the femoral artery was used to insert a bovine jugular vein valve (Melody transcatheter pulmonary valve) within the existing, regurgitant aortic bioprosthesis as valve-in-valve therapy for severe aortic insufficiency. The undeployed Melody valve can be seen within the aortic bioprosthesis. B, Postdeployment image of the Melody valve within the bioprosthesis, with mild, residual aortic insufficiency present.

with uncorrected $\mathrm{AI}^{7}$ In the present case series, 4 of the 5 patients survived redo sternotomy and experienced functional recovery. The 1 patient who died postoperatively had undergone surgery after a significant delay and had renal failure before the attempted valve procedure. Again, these examples reiterate that earlier, more elective surgical interventions could yield better outcomes, and delaying intervention to the point of end organ dysfunction will predictably yield negative outcomes. 


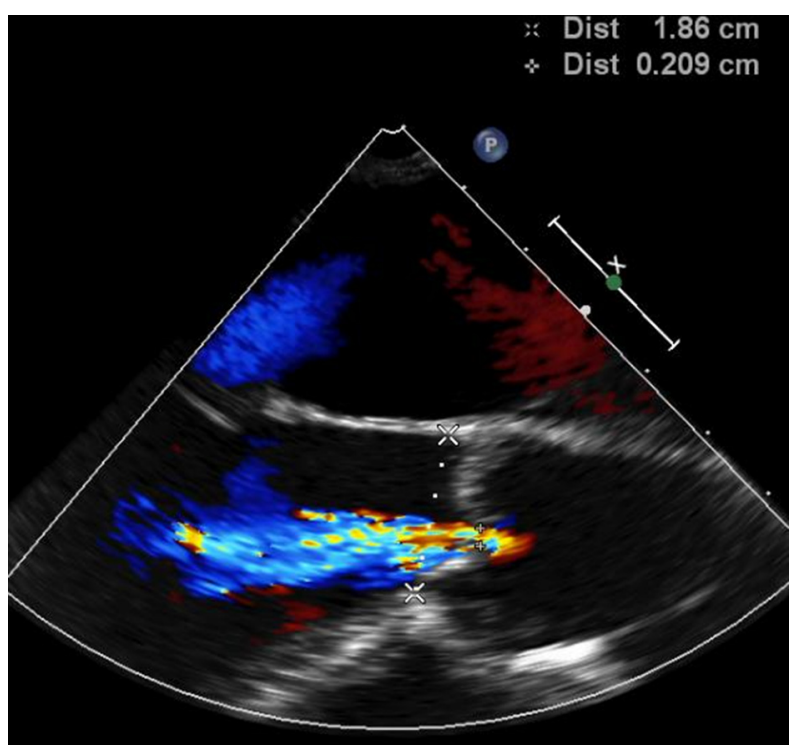

FIGURE 3. Transesophageal echocardiogram demonstrating trivial aortic insufficiency at HeartMate II left ventricular assist device implantation with grading based on the width of the regurgitant jet vena contracta. ${ }^{14}$ At present, it is unclear whether nonsignificant aortic insufficiency predisposes the patient to worsening valvular regurgitation after left ventricular assist device implantation.

One concern with the development of severe, de novo AI in the postoperative setting, particularly relatively soon after surgery, is that unrecognized, significant AI was present preoperatively. However, on examining the patients we have presented, specifically patients 3,4 , and 5 , in whom aortic valve pathologic features were noted at the subsequent operation, the intraoperative transesophageal echocardiography at LVAD implantation revealed trace, mild, and trace AI, respectively. It has been our clinical practice to perform an aortic valve intervention in the setting of AI that is greater than mild at LVAD implantation. Because none of these patients met this criterion, no aortic valve procedure was initially pursued. Examination of a similar patient population by our group revealed that preimplant AI was not a significant predictor of progression of AI after implantation. ${ }^{9}$ A review of the patients after the described interventions indicated that patients who underwent bioprosthetic AVR have prostheses that remain closed during most of the cardiac cycle, as shown by echocardiography, without any evidence of premature structural deterioration. Also, the 1 patient with bioprosthetic AVR before LVAD implantation had had the longest duration between implantation and the development of severe AI (4 years), potentially indicating that these prostheses are relatively durable when subjected to CF.

A transcatheter strategy to correct severe AI in these patients would help avoid the risks associated with redo sternotomy. Innovative approaches incorporating transcatheter aortic valve procedures during LVAD therapy have been used successfully elsewhere. ${ }^{26,27}$ In 2 of our 6 cases, a transcatheter treatment was attempted: 1 AMPLATZER VSD occluder was deployed, and 1 bovine jugular vein valve was inserted within an existing bioprosthetic aortic valve. However, the AMPLATZER occluder migrated, and we are aware of 1 other case at another institution in which device migration occurred (Laura Janney and Robert Adamson, personal communication, 2013). In our case, open surgery was required, but the patient remained stable and did not experience the need for emergency surgery. The use of a transcatheter Melody valve in 1 case was successful at reducing AI and achieving functional improvement. However, in that case, the patient had a previous bioprosthetic AVR, and the rigid struts provided a good landing zone and seating area for the transcatheter valve. The Melody valve also has sizing limitations, with the largest available prosthesis $22 \mathrm{~mm}$ in diameter. The recently approved Sapien transcatheter aortic valve (Edwards Lifesciences, Irvine, Calif) is available in $23-\mathrm{mm}$ and $26-\mathrm{mm}$ diameters, also limiting application to smaller annular diameters, and the device is not yet widely available. Finally, the CoreValve device (Medtronic) has 26-, 29-, and 31-mm sizes, but the valve remains in clinical trials. Thus, the CoreValve is not available for off-label applications such as treatment of postLVAD AI. Importantly, neither the Sapien nor the CoreValve device was designed to treat AI, but both have been used for this application on a limited basis outside of the United States. Treatment of AI using transcatheter methods appears more challenging than treating calcific aortic stenosis, because the absence of valvular calcification obscures the precise identity of the aortic annular landing zones. However, we speculate that this technique will almost certainly become the treatment of choice for significant post-LVAD AI once additional experience has been gained.

Overall, these results have indicated that the presence of severe AI after LVAD implantation, particularly in the setting of symptoms of heart failure, warrants intervention. Also, with appropriate, early treatment, patients have the potential for functional recovery. In this scenario, our typical approach would consist of aortic valve repair with a modified Park stitch; however, certain valvular characteristics, such as heavily calcified leaflets, might preclude this approach, necessitating AVR. Additionally, in cases in which redo sternotomy proves to be risk prohibitive, transcatheter interventions provide an alternative with the potential for functional recovery. Although we have demonstrated good results with these interventions, the small number of repair or replacement procedures preclude comment on the advantages of 1 technique over another, and additional investigation is warranted.

\section{CONCLUSIONS}

Surgical treatment of post-LVAD AI by aortic valve repair, valve orifice oversewing, or AVR can be effective at restoring 
functional capacity for CFLVAD patients who develop symptomatic, severe AI. Transcatheter approaches to these difficult scenarios offer less invasive options in appropriate cases.

\section{References}

1. Kirklin JK, Naftel DC, Kormos RL, Stevenson LW, Pagani FD, Miller MA, et al Third INTERMACS Annual Report: the evolution of destination therapy in the United States. J Heart Lung Transplant. 2011;30:115-23.

2. Slaughter MS, Rogers JG, Milano CA, Russell SD, Conte JV, Feldman D, et al. Advanced heart failure treated with continuous-flow left ventricular assist device. $N$ Engl J Med. 2009;361:2241-51.

3. Rao V, Slater JP, Edwards NM, Naka Y, Oz MC. Surgical management of valvular disease in patients requiring left ventricular assist device support. Ann Thorac Surg. 2001;71:1448-53.

4. Baradarian S, Dembitsky WP, Jaski B, Abolhoda A, Adamson R, Chillcot S, Daily PO. Left ventricular outflow tract obstruction associated with chronic ventricular assist device support. ASAIO J. 2002;48:665-7.

5. Letsou GV, Connelly JH, Delgado RM III, Myers TJ, Gregoric ID, Smart FW, et al. Is native aortic valve commissural fusion in patients with long-term left ventricular assist devices associated with clinically important aortic insufficiency? J Heart Lung Transplant. 2006;25:395-9.

6. Rose AG, Park SJ. Pathology in patients with ventricular assist devices: a study of 21 autopsies, 24 ventricular apical core biopsies and 24 explanted hearts. Cardiovasc Pathol. 2005;14:19-23.

7. Adamson RM, Dembitsky WP, Baradarian S, Chammas J, May-Newman K, Chillcott S, et al. Aortic valve closure associated with HeartMate left ventricular device support: technical considerations and long-term results. J Heart Lung Transplant. 2011;30:576-82.

8. Mudd JO, Cuda JD, Halushka M, Soderlund KA, Conte JV, Russell SD. Fusion of aortic valve commissures in patients supported by a continuous axial flow left ventricular assist device. J Heart Lung Transplant. 2008;27:1269-74.

9. Rajagopal K, Daneshmand MA, Patel CB, Ganapathi AM, Schechter MA, Rogers JG, et al. Natural history and clinical effect of aortic valve regurgitation after left ventricular assist device implantation. J Thorac Cardiovasc Surg. 2013; 145:1373-9.

10. Cowger J, Pagani FD, Haft JW, Romano MA, Aaronson KD, Kolias TJ. The development of aortic insufficiency in left ventricular assist device-supported patients. Circ Heart Fail. 2010;3:668-74

11. Pak SW, Uriel N, Takayama H, Cappleman S, Song R, Colombo PC, et al. Prevalence of de novo aortic insufficiency during long-term support with left ventricular assist devices. J Heart Lung Transplant. 2010;29:1172-6.

12. Toda K, Fujita T, Domae K, Shimahara Y, Kobayashi J, Nakatani T. Late aortic insufficiency related to poor prognosis during left ventricular assist device support. Ann Thorac Surg. 2011;92:929-34.

13. Park SJ, Liao KK, Segurola R, Madhu KP, Miller LW. Management of aortic insufficiency in patients with left ventricular assist devices: a simple coaptation stitch method (Park's stitch). J Thorac Cardiovasc Surg. 2004;127:264-6.
14. Frazier OH. Unforeseen consequences of therapy with continuous-flow pumps. Circ Heart Fail. 2010;3:647-9.

15. Jakovljevic DG, George RS, Nunan D, Donovan G, Bougard RS, Yacoub $\mathrm{MH}$, et al. The impact of acute reduction of continuous-flow left ventricular assist device support on cardiac and exercise performance. Heart. 2010;96:1390-5.

16. Pagani FD, Miller LW, Russell SD, Aaronson KD, John R, Boyle AJ, et al. Extended mechanical circulatory support with a continuous-flow rotary left ventricular assist device. J Am Coll Cardiol. 2009;54:312-21.

17. John R, Boyle A, Pagani F, Miller L. Physiologic and pathologic changes in patients with continuous-flow ventricular assist devices. J Cardiovasc Transl Res. 2009;2:154-8.

18. John R, Kamdar F, Eckman P, Colvin-Adams M, Boyle A, Shumway S, et al. Lessons learned from experience with over 100 consecutive HeartMate II left ventricular assist devices. Ann Thorac Surg. 2011;92:1593-9; discussion $1599-600$.

19. Zamarripa Garcia MA, Enriquez LA, Dembitsky W, May-Newman K. The effect of aortic valve incompetence on the hemodynamics of a continuous flow ventricular assist device in a mock circulation. ASAIO J. 2008;54: 237-44.

20. Hatano M, Kinugawa K, Shiga T, Kato N, Endo M, Hisagi M, et al. Less frequent opening of the aortic valve and a continuous flow pump are risk factors for postoperative onset of aortic insufficiency in patients with a left ventricular assist device. Circ J. 2011;75:1147-55.

21. May-Newman K, Hillen B, Dembitsky W. Effect of left ventricular assist device outflow conduit anastomosis location on flow patterns in the native aorta. ASAIO J. 2006;52:132-9.

22. May-Newman K, Mendoza A, Abulon DJ, Joshi M, Kunda A, Dembitsky W. Geometry and fusion of aortic valves from pulsatile flow ventricular assist device patients. J Heart Valve Dis. 2011;20:149-58.

23. May-Newman K, Enriquez-Almaguer L, Posuwattanakul P, Dembitsky W. Biomechanics of the aortic valve in the continuous flow VAD-assisted heart. ASAIO J. 2010;56:301-8.

24. Klotz S, Burkhoff D, Garrelds IM, Boomsma F, Danser AH. The impact of left ventricular assist device-induced left ventricular unloading on the myocardial renin-angiotensin-aldosterone system: therapeutic consequences? Eur Heart J. 2009;30:805-12.

25. Klotz S, Danser AH, Foronjy RF, Oz MC, Wang J, Mancini D, et al. The impact of angiotensin-converting enzyme inhibitor therapy on the extracellular collagen matrix during left ventricular assist device support in patients with end-stage heart failure. J Am Coll Cardiol. 2007;49:1166-74.

26. Grohmann J, Blanke P, Benk C, Schlensak C. Trans-catheter closure of the native aortic valve with an Amplatzer occluder to treat progressive aortic regurgitation after implantation of a left-ventricular assist device. Eur J Cardiothorac Surg. 2011;39:e181-3.

27. Menon AK, Dohmen G, Mahnken AH, Autschbach R. Successful combined procedure of HeartMate II left ventricular assist device implantation and minimally invasive transapical aortic valve replacement. J Thorac Cardiovasc Surg. 2011;142:708-9. 